\title{
Gender Differences in 1-Year Clinical Characteristics and Outcomes after Stroke: Results from the China National Stroke Registry
}

\author{
Zhan Wang ${ }^{19}$, Jingjing $\mathrm{Li}^{19}$, Chunxue Wang ${ }^{1}$, Xiaomei Yao ${ }^{2}$, Xingquan Zhao', Yilong Wang ${ }^{1}$, Hao $\mathrm{Li}^{1}$, \\ Gaifen Liu' ${ }^{1}$, Anxin Wang ${ }^{1}$, Yongjun Wang ${ }^{1 *}$
}

1 Department of Neurology, Beijing Tiantan Hospital, Capital Medical University, Beijing, China, 2 Department of Oncology, McMaster University, Ontario, Canada

\begin{abstract}
Background: Previous reports have shown inconsistent results on clinical outcomes between women and men after stroke, and little is known about gender differences on outcomes in Chinese post-stroke patients. The aim of this study was to explore whether there were gender differences on clinical characteristics and outcomes in Chinese patients after ischemic stroke by using the data from the China National Stroke Registry (CNSR).

Methods and Findings: Out of 12,415 consecutively recruited patients with acute ischemic stroke in the CNSR from 2007 to $2008,11,560$ (93.1\%) patients were followed up for 12 months. Their clinical characteristics and outcomes on death, recurrence, and dependency were recorded. The multivariate logistic regression was performed to determine whether there were gender differences in these outcomes. Women were older than men at baseline $(67.9$ vs. 64.0 years, $P<0.001)$. Women had a higher mortality, recurrence rate, and dependency rate at 3, 6, and 12 months than men, but after adjusting for age, history of diabetes, pre-stroke dependency, stroke severity, in-hospital complications, and other confounders, there were no statistically significant differences in gender on mortality and recurrence rate at 3, 6, and 12 months; and dependency rate at 3 , and 6 months. However, the dependency rate at 12 months remained significantly higher in women (odds ratio, 1.24; $95 \%$ confidence interval, 1.06 to 1.45 ).

Conclusions: There are many differences in clinical characteristics between women and men after ischemic stroke in China. Compared with men, women are more dependent at 12 months after stroke. This difference still exists after controlling the potential confounders.
\end{abstract}

Citation: Wang Z, Li J, Wang C, Yao X, Zhao X, et al. (2013) Gender Differences in 1-Year Clinical Characteristics and Outcomes after Stroke: Results from the China National Stroke Registry. PLoS ONE 8(2): e56459. doi:10.1371/journal.pone.0056459

Editor: Weili Zhang, FuWai Hospital, Chinese Academy of Medical Sciences, China

Received April 17, 2012; Accepted January 14, 2013; Published February 13, 2013

Copyright: () 2013 Wang et al. This is an open-access article distributed under the terms of the Creative Commons Attribution License, which permits unrestricted use, distribution, and reproduction in any medium, provided the original author and source are credited.

Funding: This study was funded by the Ministry of Science and Technology and the Ministry of Health of the People's Republic of China and the Grant No. is National S \& T Major Project of China (2011BAI08B02) and by the Research Special Fund For Public Welfare Industry of Health (200902004). Grants were received from State Key Development, Program of Basic Research of China [2009CB521905], the National Science Foundation (grant no. 81071115), and the Beijing Science and Technology Committee (grant no. 7102050). The funders had no role in study design, data collection and analysis, decision to publish, or preparation of the manuscript.

Competing Interests: The authors have declared that no competing interests exist.

* E-mail: snow_sen@yahoo.com

9 These authors contributed equally to this work.

\section{Introduction}

Stroke is one of the leading causes of death and disability throughout the world [1]. The incidence and burden of stroke in China is increasing rapidly over time just like in other developing countries. It is now becoming the first leading cause of death in China [2]. Gender differences on prognostic outcomes in stroke patients have been explored in many countries. However, the results from these studies are inconsistent. Some studies showed that women had poorer prognoses $[3,4,5,6,7]$, but some studies reported no gender differences on survival rate [8,9], and others even demonstrated that women had better outcomes than men after stroke $[10,11,12]$. These differences among the previous studies may be relevant to the different patient selection criteria and follow-up periods (most studies had a less than one-year follow-up). Furthermore, most studies included patients with all types of stroke (i.e., ischemic stroke, intracerebral hemorrhage, and subarachnoid hemorrhage [SAH]), which would make it hard to interpret the results because gender differences already exist in the different types of stroke.

It has been realized that very few studies have investigated the gender differences on stroke in Chinese patients, especially for the prognostic outcomes [13]. The China National Stroke Registry (CNSR) was a population-based, large-scale prospective registry, which covered all 27 provinces and 4 municipalities in China including the Hong Kong region. The more details of the study design for the CNSR have been published elsewhere [14]. As a part of the GNSR, this study only focused on patients with ischemic stroke and with completed follow-up data for one year. The purpose of this study was to determine whether there were gender differences on clinical characteristics and 3-, 6- and 12month outcomes in Chinese patients after ischemic stroke. 


\section{Materials and Methods}

\section{Subjects}

The CNSR prospectively recruited consecutive stroke patients from 132 tertiary or secondary hospitals in China from September 2007 to August 2008. Patients were eligible to enroll if they were 18 years of age or older with stroke symptom onset within 14 days, and diagnosed as stroke or transient ischemic attack (TIA). Stroke was defined as acute ischemic stroke, intracerebral hemorrhage, and $\mathrm{SAH}$, with brain computed tomography (CT) or magnetic resonance (MR) confirmation, which was consistent with the diagnostic criteria from World Health Organization [15]. Except for the patients who refused to participate in the survey, a total of 22,216 patients were enrolled in the GNSR and 14,526 of them had ischemic stroke. Among 14,526 patients with ischemic stroke, 816 patients who were transferred from other non-enrolled hospitals were excluded from our study because the treatments in other hospitals would affect the prognosis of stroke. Also, 1,295 patients who refused to be followed up were excluded from our study. Thus, 12,415 patients with ischemic stroke who had completed baseline information at the first visit were included in our study. After a one-year follow-up, the completed data from 11,560 patients $(93.1 \%)$ were obtained (Figure 1). The study was approved by the ethics committees at all participating hospitals and the written informed consents were obtained from all participants or their designated relatives.

\section{Patient characteristics}

In the current study, patient characteristics information at admission was collected, including age, sex, pre-stroke dependency, social-economic factors, and potential risk factors for stroke.
The pre-stroke dependency was defined as the modified Rankin Scale $(\mathrm{mRS})$ score of 3-5. The social-economic factors included marital status, patients' living arrangement before admission, education, and health insurance. The marital status included two types: married, and single/divorced/widowed/remarried. The patients' living arrangements were grouped into three categories: living with other family members, alone, and in a nursing home. The educational background was grouped into three types: elementary or below, middle school, and high school or above. The patients' health insurance was classified into four groups based on the national conditions in China $[16,17,18]$ : (1) the Basic Health Insurance Scheme for the employed urban residents, which was jointly funded by the government, employers, and employees; (2) the New Cooperative Medical System provided by the Chinese government for rural residents is a communal system aiming at major medical treatment; (3) self-paid (no insurance), and (4) commercial or other insurances.

The potential risk factors included body mass index (BMI, calculated as weight in kilograms $[\mathrm{kg}]$ divided by height in meters squared $\left[\mathrm{m}^{2}\right]$ ); smoking status (smokers were defined as patients who were previous or present smokers); moderate or heavy alcohol (defined as more than 2 standard alcohol consumption per day [1 standard alcohol consumption equal to $120 \mathrm{ml}$ wine, $360 \mathrm{ml}$ beer, or $45 \mathrm{ml}$ white spirit]). The potential risk factors also included the common risk factors of ischemic stroke, including the history of hypertension (defined as a self-reported history or anti-hypertensive drug use), diabetes mellitus (a self-reported history or current hypoglycemic medications use), dyslipidemia (a self-reported history or lipid-lowering drug use), coronary heart disease (a selfreported history or an angina history confirmed by a cardiologist in prior clinic visits), atrial fibrillation (a self-reported history with

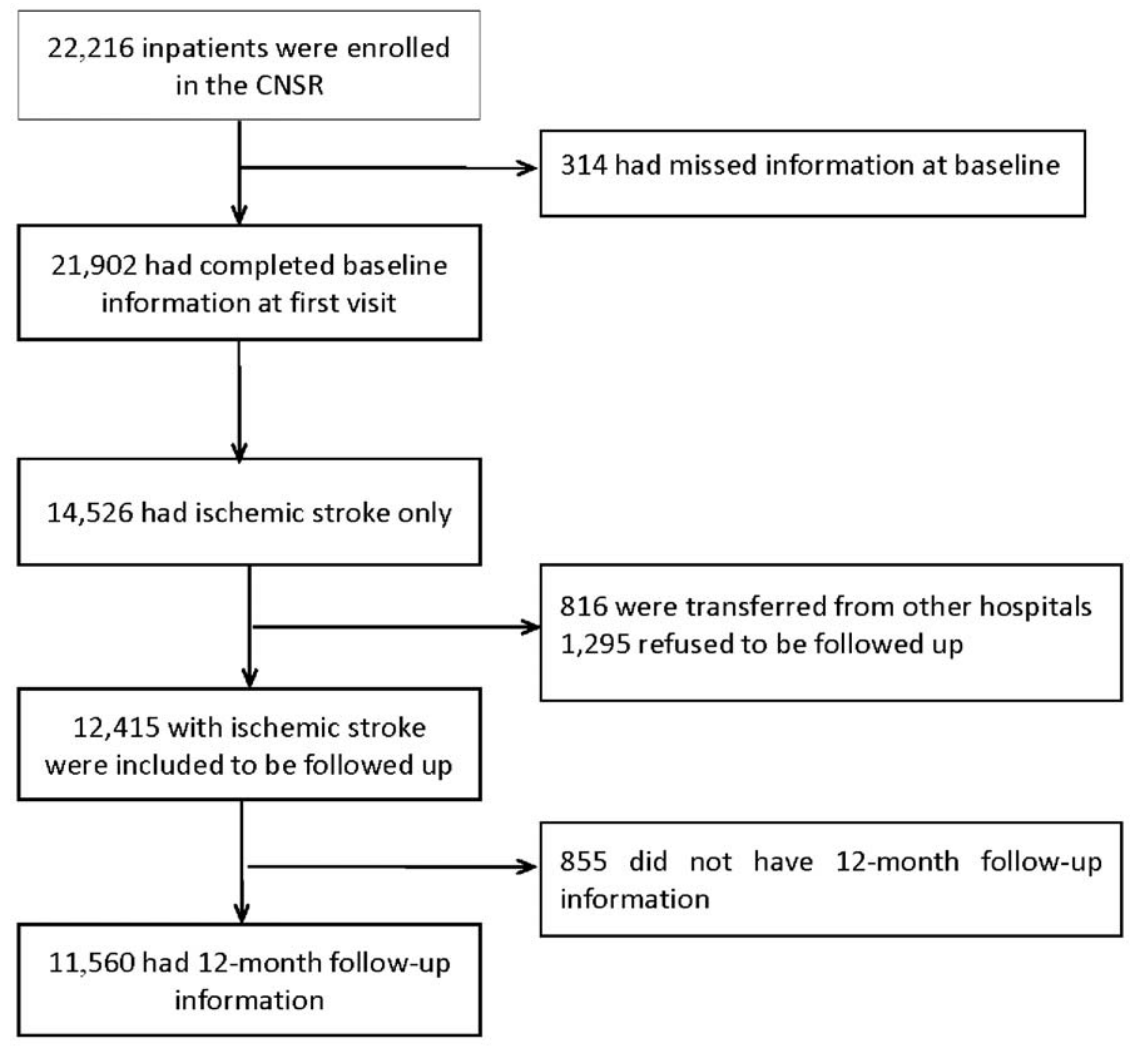

Figure 1. Flow diagram of participants. doi:10.1371/journal.pone.0056459.g001 
at least one confirmed electrocardiogram), and prior stroke (medical chart confirmed).

Additionally, the blood glucose, systolic and diastolic blood pressure (SBP and DBP), and the stroke severity (measured by the National Institute of Health Stroke Scale [NIHSS] [19] and Glasgow Coma Scale [GCS] [20]) at admission; the in-hospital complications; and thrombolysis treatment within three hours from the beginning of stroke symptom were also recorded.

\section{Outcomes}

Death, recurrence, and dependency at 3, 6, and 12 months after stroke were assessed through telephone follow-ups. Recurrence was self-reported and defined as the aggravation of the original symptoms, new signs, or re-hospitalization with the diagnosis of ischemic stroke, intracerebral hemorrhage, or SAH. For patients who admitted hospitals again due to recurrent stroke, the information of diagnosis and classification of recurrent stroke were obtained from contacting the discharge department in the hospitals. Dependency was defined as the mRS score of 3-5. Mortality was defined as the cumulative death rate at 3, 6, and 12 months from the onset of stroke for any reason of death. The recurrence and dependency rates were defined as the cumulative rates at 3, 6, and 12 months after ischemic stroke, respectively. The standard scripts were used to collect the data of outcomes by the trained research personnel at Beijing Tiantan Hospital. The enrolled patients were the targeted respondents for the questionnaire. When it was impossible to talk with an enrolled patient or when the interviewer considered that the information provided by the patient was unreliable, his/her care-giver was contacted and interviewed. More details about the outcome assessment can be found in the protocol of the GNSR [14].

\section{Statistical analysis}

The $\mathrm{t}$ test, Wilcoxon test, and chi-square test were used for univariate comparisons between genders, continuous variables in normal and abnormal distribution, and the categorical variables, respectively. The multivariate logistic regression was used to evaluate whether any of the patient characteristics (mentioned in the last section) might affect mortality, recurrence rate, and dependency rate at 12 months after onset of stroke. All data were analyzed using SAS version 9.2 statistical software and a two-sided significance level of $\alpha=0.05$ was assumed. The data analysts were blinded to the patients' gender information.

\section{Results}

\section{Clinical characteristics between enrolled and excluded patients with ischemic stroke}

Table 1 shows the baseline characteristics of the 11,560 enrolled patients and 2,966 excluded patients. The comparative results demonstrated that there was no significant difference in gender $(61.6 \%$ versus $63.4 \%, P=0.070)$, but the enrolled patients were two years older than the excluded patients on average (67.0 versus 65.0 years, $P<0.001)$. The enrolled patients had a higher percentage of having a history of hypertension, dyslipidemia, prior stroke, atrial fibrillation, or coronary heart disease; a lower percentage of being married; a higher percentage of Basic Health Insurance Scheme; and a higher percentage of complications of new stroke, new atrial fibrillation, pneumonia, and stress ulcer than the excluded patients. There was no statistical difference for the severity of stroke between the enrolled and excluded patients.

\section{Clinical characteristics between women and men}

Among 11,560 ischemic stroke patients, 4442 (38.4\%) were women. On average, women were 3.9 years older than men (mean 67.9 versus 64.0 years, $P<0.001)$ in Table 2 . Before stroke, women lived alone more $(4.7 \%$ versus $2.8 \%, P<0.001)$, were single or divorced more $(16.4 \%$ versus $6.5 \%, P<0.001)$, had a higher proportion of self-paid insurance $(23.4 \%$ versus $16.3 \%, P<0.001)$ and a lower educational level $(62.2 \%$ versus $35.6 \%, P<0.001)$ than men. Compared with men, women also had a higher proportion of pre-stroke dependency $(6.0 \%$ versus $4.6 \%, P<0.001)$.

Women were significantly more likely to have histories of hypertension, diabetes, coronary heart disease, and atrial fibrillation than men, and had a significantly higher frequency of overweight $\left(6.7 \%\right.$ versus $4.6 \%$ for $\left.\mathrm{BMI} \geq 30 \mathrm{~kg} / \mathrm{m}^{2}\right)$, but were significantly less likely to smoke and/or to have moderate or heavy alcohol. The level of blood glucose at admission was higher in women than in men as well as DBP, but SBP was not significantly different between them.

Table 2 also shows that women had severer stroke and were more likely to be comatose than men. There were no gender differences in the proportion of patients with thrombolysis treatment $(4.2 \%$ versus $4.5 \%$ for women versus men, $P=0.337$ ), and in the proportion of patients presenting to hospital within three hours of symptom recognition $(22.3 \%$ versus $21.2 \%$, $P=0.188$ ). During the hospitalization, women were significantly more likely to experience seizure, urinary tract infection, pneumonia, decubitus ulcer, and new atrial fibrillation.

\section{Outcomes at 3, 6, and 12 months after stroke onset}

Without controlling potential confounders, the outcomes of mortality, recurrence rate, and dependency rate at 3, 6, and 12 months after stroke are shown in Table 3 . The multivariate logistic regression analysis showed that age, history of diabetes, history of stroke, pre-stroke dependence, stroke severity, blood glucose at admission, and the complications of pneumonia and new atrial fibrillation had significant association with mortality, recurrence rate, and dependency rate at 12 months (Table 4). The mortality was significantly higher in post-stroke women than those in men at 3,6 , and 12 months, respectively in Table $3(11.3 \%$ versus $7.6 \%$, $14.4 \%$ versus $9.7 \%, 17.8 \%$ versus $12.3 \%$; all $P<0.001$ ), but after adjusting for the above confounding factors, there were no gender differences on mortality at 3, 6, and 12 months in Table 5 (adjusted odds ratio [OR] 1.06, 1.10, 1.09, respectively; all $P>0.05)$.

A similar pattern was observed for recurrence rate. Before the adjustment of the above confounding factors, women's recurrence rates were significantly higher than men's at 3,6, and 12 months after stroke onset $(14.4 \%$ versus $12.0 \%, 18.1 \%$ versus $14.7 \%$, $19.9 \%$ versus $16.4 \%$, respectively, all $P<0.001$ in Table 3$)$. There were no more significant differences between them after the adjustment (adjusted OR 1.03, 1.06, 1.05, respectively; all $P>0.05$ in Table 5).

The unadjusted dependency rates were significantly higher in women than those in men at 3, 6, 12 months after stroke $34.1 \%$ versus $24.4 \%, 30.7 \%$ versus $22.4 \%, 28.7 \%$ versus $19.1 \%$, respectively; all $P<0.001$ in Table 3 ) and no more significant differences were observed after adjusting for the confounders (adjusted OR 1.15, 1.13, respectively; all $P>0.05$ in Table 5). However, women were more likely to be dependent than men at 12 months even after adjusting for the confounders (adjusted OR, $1.24 ; 95 \%$ confidence interval $[\mathrm{CI}], 1.06$ to $1.45, P<0.05$ in Table 5). 
Table 1. Baseline characteristics of enrolled and excluded patients with ischemic stroke.

\begin{tabular}{|c|c|c|c|c|}
\hline \multicolumn{2}{|l|}{ Variables } & \multirow{2}{*}{$\begin{array}{l}\begin{array}{l}\text { Enrolled patients } \\
(\mathbf{n}=\mathbf{1 1}, \mathbf{5 6 0})\end{array} \\
7,118(61.6 \%)\end{array}$} & \multirow{2}{*}{$\begin{array}{l}\begin{array}{l}\text { Excluded patients* } \\
(\mathbf{n}=\mathbf{2 , 9 6 6 )}\end{array} \\
1,880(63.4 \%)\end{array}$} & \multirow{2}{*}{$\begin{array}{l}P \text { Value } \\
0.070\end{array}$} \\
\hline Gender & men & & & \\
\hline & women & 4,442 (38.4\%) & $1,086(36.6 \%)$ & \\
\hline \multicolumn{2}{|c|}{ Mean age (range), years } & $67.0(57.0-75.0)$ & $65.0(54.0-74.0)$ & $<0.001$ \\
\hline \multicolumn{2}{|c|}{ Pre-stroke dependency } & $595(5.1 \%)$ & $148(5.0 \%)$ & 0.729 \\
\hline \multicolumn{5}{|c|}{ Social-economic factors: } \\
\hline \multirow[t]{2}{*}{ marital status } & married & $10,320(89.7 \%)$ & 2,677 (91.1\%) & 0.020 \\
\hline & single/divorced/widowed/remarried & 1,185 (10.3\%) & $260(8.9 \%)$ & \\
\hline \multirow[t]{3}{*}{ education } & elementary or below & $5,300(45.9 \%)$ & $1,246(45.1 \%)$ & 0.112 \\
\hline & middle school & $2,976(25.7 \%)$ & 764 (27.6\%) & \\
\hline & high school or above & $3,284(28.4 \%)$ & $754(27.3 \%)$ & \\
\hline \multirow[t]{4}{*}{ health insurance } & Basic Health Insurance Scheme & 7,027 (60.8\%) & $1,496(50.4 \%)$ & $<0.001$ \\
\hline & New Cooperative Medical System & $1,953(16.9 \%)$ & $629(21.2 \%)$ & \\
\hline & self-paid & $378(3.3 \%)$ & 129 (4.3\%) & \\
\hline & others (including commercial) & 2,202 (19.1\%) & $712(24.0 \%)$ & \\
\hline \multicolumn{5}{|c|}{ Risk factors of stroke: } \\
\hline \multirow[t]{3}{*}{ BMI at admission } & $\mathrm{BMl}<25\left(\mathrm{~kg} / \mathrm{m}^{2}\right)$ & 6,322 (60.6\%) & $1,505(63.5 \%)$ & 0.031 \\
\hline & $25 \leq \mathrm{BMI}<30\left(\mathrm{~kg} / \mathrm{m}^{2}\right)$ & 3,537 (34.0\%) & $741(31.3 \%)$ & \\
\hline & $\mathrm{BMI} \geq 30\left(\mathrm{~kg} / \mathrm{m}^{2}\right)$ & $559(5.4 \%)$ & $124(5.2 \%)$ & \\
\hline \multicolumn{2}{|l|}{ smoking } & 4,586 (39.7\%) & $1,187(40.7 \%)$ & 0.295 \\
\hline \multicolumn{2}{|c|}{ moderate or heavy alcohol } & $1,082(9.4 \%)$ & $278(9.4 \%)$ & 0.983 \\
\hline \multicolumn{2}{|c|}{ history of hypertension } & $7,401(64.0 \%)$ & $1,786(60.2 \%)$ & $<0.001$ \\
\hline \multicolumn{2}{|c|}{ history of diabetes mellitus } & $2,503(21.6 \%)$ & $595(20.1 \%)$ & 0.059 \\
\hline \multicolumn{2}{|c|}{ history of dyslipidemia } & $1,312(11.3 \%)$ & $248(8.4 \%)$ & $<0.001$ \\
\hline \multicolumn{2}{|c|}{ history of coronary heart disease } & $1,685(14.6 \%)$ & 331 (11.2\%) & $<0.001$ \\
\hline \multicolumn{2}{|c|}{ history of atrial fibrillation } & $875(7.6 \%)$ & $169(5.7 \%)$ & $<0.001$ \\
\hline \multicolumn{2}{|l|}{ history of prior stroke } & 3,967 (34.3\%) & 894 (30.1\%) & $<0.001$ \\
\hline \multicolumn{5}{|l|}{ Stroke severity: } \\
\hline \multirow[t]{3}{*}{ NIHSS at admission } & $0-4$ & $5,734(49.6 \%)$ & $1,477(49.8 \%)$ & 0.062 \\
\hline & $5-14$ & 4,384 (37.9\%) & $1,164(39.2 \%)$ & \\
\hline & $\geq 15$ & $1,442(12.5 \%)$ & $325(11.0 \%)$ & \\
\hline \multirow[t]{4}{*}{ GCS at admission } & $=15$ & 7,459 (69.1\%) & 1,963 (71.3\%) & 0.070 \\
\hline & 13-14 & $1,083(10.0 \%)$ & $273(9.9 \%)$ & \\
\hline & $9-12$ & $1,187(11.0 \%)$ & $284(10.3 \%)$ & \\
\hline & $3-8$ & $1,070(9.9 \%)$ & $234(8.5 \%)$ & \\
\hline \multicolumn{5}{|c|}{ In-hospitalization complications: } \\
\hline new stroke & & $452(3.9 \%)$ & $50(1.7 \%)$ & $<0.001$ \\
\hline seizure & & $131(1.1 \%)$ & $26(1.0 \%)$ & 0.312 \\
\hline urinary tract infectio & & $457(3.9 \%)$ & $87(2.9 \%)$ & 0.009 \\
\hline pneumonia & & $1,373(11.9 \%)$ & $275(9.3 \%)$ & $<0.001$ \\
\hline gastrointestinal hem & & $317(2.7 \%)$ & $42(1.4 \%)$ & $<0.001$ \\
\hline decubitus ulcer & & $93(0.8 \%)$ & $20(0.7 \%)$ & 0.584 \\
\hline deep venous throm & & $51(0.4 \%)$ & $12(0.4 \%)$ & 0.787 \\
\hline pulmonary embolisr & & $39(0.3 \%)$ & $13(0.4 \%)$ & 0.412 \\
\hline myocardial infarct & & $75(0.7 \%)$ & $16(0.5 \%)$ & 0.501 \\
\hline atrial fibrillation & & $899(7.8 \%)$ & $175(5.9 \%)$ & $<0.001$ \\
\hline
\end{tabular}

BMI: body mass index, NIHSS: National Institute of Health Stroke Scale, GCS: Glasgow Coma Scale.

*The excluded patients with ischemic stroke included: 816 patients who were transferred from the other hospitals, 1295 patients who refused to participate in the follow-ups, and 855 patients who were lost to follow-up.

doi:10.1371/journal.pone.0056459.t001 
Table 2. Baseline characteristics of different gender patients with ischemic stroke.

\begin{tabular}{|c|c|c|c|c|}
\hline \multicolumn{2}{|l|}{ Variables } & Women $(n=4,442)$ & Men $(n=7,118)$ & $P$ Value \\
\hline \multicolumn{2}{|l|}{$\operatorname{Age}(X \pm S D)(y e a r s)$} & $67.9 \pm 11.9$ & $64.0 \pm 12.3$ & $<0.001$ \\
\hline \multicolumn{2}{|c|}{ Pre-stroke dependency } & $267(6.0 \%)$ & $328(4.6 \%)$ & $<0.001$ \\
\hline \multicolumn{5}{|c|}{ Social-economic factors: } \\
\hline \multirow[t]{2}{*}{ marital status } & married & $3,691(83.6 \%)$ & $6,629(93.5 \%)$ & $<0.001$ \\
\hline & single/divorced/widowed/remarried & 725 (16.4\%) & $460(6.5 \%)$ & \\
\hline \multirow[t]{3}{*}{ living arrangement } & with other family members & $4,160(94.7 \%)$ & $6,850(97.0 \%)$ & $<0.001$ \\
\hline & alone & $208(4.7 \%)$ & $197(2.8 \%)$ & \\
\hline & nursing home & $26(0.6 \%)$ & $18(0.2 \%)$ & \\
\hline \multirow[t]{3}{*}{ education } & elementary or below & $2,763(62.2 \%)$ & $2,537(35.6 \%)$ & $<0.001$ \\
\hline & middle school & $878(19.8 \%)$ & $2,098(29.5 \%)$ & \\
\hline & high school or above & $801(18.0 \%)$ & $2,483(34.9 \%)$ & \\
\hline \multirow[t]{4}{*}{ health insurance } & Basic Health Insurance Scheme & $2,339(52.7 \%)$ & $4,688(65.9 \%)$ & $<0.001$ \\
\hline & New Cooperative Medical System & $893(20.1 \%)$ & $1,060(14.9 \%)$ & \\
\hline & self-paid & $1,040(23.4 \%)$ & $1,162(16.3 \%)$ & \\
\hline & others (including commercial) & $170(3.8 \%)$ & $208(2.9 \%)$ & \\
\hline \multicolumn{5}{|c|}{ Risk factors of stroke: } \\
\hline \multirow[t]{3}{*}{ BMI at admission } & $\mathrm{BMI}<25\left(\mathrm{~kg} / \mathrm{m}^{2}\right)$ & $2,358(59.5 \%)$ & $3,964(61.4 \%)$ & $<0.001$ \\
\hline & $25 \leq \mathrm{BMl}<30\left(\mathrm{~kg} / \mathrm{m}^{2}\right)$ & $1,342(33.8 \%)$ & $2,195(34.0 \%)$ & \\
\hline & $\mathrm{BMI} \geq 30\left(\mathrm{~kg} / \mathrm{m}^{2}\right)$ & $266(6.7 \%)$ & $293(4.6 \%)$ & \\
\hline \multicolumn{2}{|l|}{ smoking } & $268(6.0 \%)$ & $4,318(60.7 \%)$ & $<0.001$ \\
\hline \multicolumn{2}{|c|}{ moderate or heavy alcohol } & $22(0.5 \%)$ & $1,060(14.9 \%)$ & $<0.001$ \\
\hline \multicolumn{2}{|c|}{ history of hypertension } & $3,026(68.1 \%)$ & $4,375(61.5 \%)$ & $<0.001$ \\
\hline \multicolumn{2}{|c|}{ history of diabetes mellitus } & $1,102(24.8 \%)$ & $1,401(19.7 \%)$ & $<0.001$ \\
\hline \multicolumn{2}{|c|}{ history of dyslipidemia } & $490(11.0 \%)$ & $822(11.5 \%)$ & 0.394 \\
\hline \multicolumn{2}{|c|}{ history of coronary heart disease } & 769 (17.3\%) & $916(12.9 \%)$ & $<0.001$ \\
\hline \multicolumn{2}{|c|}{ history of atrial fibrillation } & $494(11.1 \%)$ & $381(5.3 \%)$ & $<0.001$ \\
\hline \multicolumn{2}{|l|}{ history of prior stroke } & 1,509 (34.0\%) & $2,458(34.5 \%)$ & 0.537 \\
\hline \multicolumn{2}{|c|}{ Blood glucose at admission $(\mathrm{X} \pm \mathrm{SD})(\mathrm{mmol} / \mathrm{L})$} & $6.94 \pm 2.43$ & $6.67 \pm 2.30$ & $<0.001$ \\
\hline \multicolumn{2}{|c|}{ SBP at admission $(\mathrm{X} \pm \mathrm{SD})(\mathrm{mmHg})$} & $151.7 \pm 24.9$ & $150.1 \pm 23.7$ & 0.057 \\
\hline \multicolumn{2}{|c|}{ DBP at admission $(\mathrm{X} \pm \mathrm{SD})(\mathrm{mmHg})$} & $86.9 \pm 13.9$ & $88.5 \pm 14.0$ & $<0.001$ \\
\hline \multicolumn{5}{|l|}{ Stroke severity: } \\
\hline NIHSS at admission & $0-4$ & $2,011(45.3 \%)$ & $3,723(52.3 \%)$ & $<0.001$ \\
\hline & $5-14$ & $1,736(39.1 \%)$ & $2,648(37.2 \%)$ & \\
\hline & $\geq 15$ & $695(15.6 \%)$ & 747 (10.5\%) & \\
\hline GCS at admission & $=15$ & 2,757 (66.3\%) & 4,702 (70.8\%) & $<0.001$ \\
\hline & $13-14$ & $415(10.0 \%)$ & $668(10.1 \%)$ & \\
\hline & $9-12$ & $504(12.1 \%)$ & $683(10.3 \%)$ & \\
\hline & $3-8$ & 485 (11.7\%) & $585(8.8 \%)$ & \\
\hline Onset to arrival ti & & $840(22.3 \%)$ & 1,279 (21.2\%) & 0.188 \\
\hline Thrombolysis give & & $156(4.2 \%)$ & 269 (4.5\%) & 0.337 \\
\hline In-hospitalization & & & & \\
\hline new stroke & & $190(4.3 \%)$ & $262(3.7 \%)$ & 0.108 \\
\hline seizure & & $63(1.4 \%)$ & $68(1.0 \%)$ & 0.022 \\
\hline urinary tract infectio & & $285(6.4 \%)$ & $172(2.4 \%)$ & $<0.001$ \\
\hline pneumonia & & $578(13.0 \%)$ & 795 (11.2\%) & 0.003 \\
\hline gastrointestinal hem & & $115(2.6 \%)$ & $202(2.8 \%)$ & 0.425 \\
\hline decubitus ulcer & & $51(1.2 \%)$ & $42(0.6 \%)$ & 0.001 \\
\hline deep venous thromb & & $26(0.6 \%)$ & $25(0.4 \%)$ & 0.065 \\
\hline
\end{tabular}


Table 2. Cont.

\begin{tabular}{llll}
\hline Variables & Women (n=4,442) & Men (n=7,118) & $\boldsymbol{P}$ Value \\
\hline pulmonary embolism & $19(0.4 \%)$ & $20(0.3 \%)$ & 0.186 \\
myocardial infarct & $33(0.7 \%)$ & $42(0.6 \%)$ & 0.319 \\
atrial fibrillation & $523(11.8 \%)$ & $376(5.3 \%)$ & $<0.001$ \\
\hline
\end{tabular}

X: mean, SD: standard deviation, BMI: body mass index, SBP: systolic blood pressure, DBP: diastolic blood pressure, NIHSS: National Institute of Health Stroke Scale, GCS: Glasgow Coma Scale.

doi:10.1371/journal.pone.0056459.t002

\section{Discussion}

The GNSR was a prospective stroke registry that had extensive coverage in China and involved the maximal stroke patients in China so far. In the current study, patients with ischemic stroke from the CNSR were selected, and the gender differences on the outcomes of mortality, recurrence rate and dependency rate were analyzed. Several previous studies [13,21,22,23,24] reported inconsistent results of the gender differences on prognoses in patients with ischemic stroke. Therefore, it prompted us to focus on the gender differences on prognoses in patients with ischemic stroke only in this study. Moreover, many previous studies $[3,4,6,8,9,11,25,26]$ aiming at gender differences in patients with all types of stroke demonstrated that there were gender differences in different stroke types. For example, the results in the Sheikh et al study [10] showed that after adjusting for age, there was no sex difference in 1-year mortality between men and women with hemorrhagic stroke (including subarachnoid hemorrhage and intracerebral hemorrhage), but men had a higher mortality than women with ischemic stroke after 1-year follow-up. Thus, we put great effort to study the gender differences on prognoses in the subgroup of ischemic stroke patients.

Among the enrolled patients with ischemic stroke, we found that women were more likely to have risk factors of stroke that is different from some published literatures in Chinese populations. For example, the 2007-2008 China National Diabetes and Metabolic Disorders Study [27] reported that the prevalences of hypertension and diabetes were higher in men than those in women who were in the primary prevention (patients had risk factors but did not develop stroke). Differing from this kind of study, our study mainly focused on patients with ischemic stroke. There were also a few studies that demonstrated all of the risk factors of stroke were significantly higher in men than in women with stroke. For instance, the Zhang et al study [28] found that young men with stroke (aged 18-45 years) were more likely to have hypertension, diabetes mellitus, and hyperlipidemia than women. However, the enrolled patients in our study were older (67.9 years for women and 64.0 years for men) than those in the Zhang et al study. The different results in risk factors could be explained by the differences in age and target populations. Similar to our findings, the Kong et al [13] and the Huang et al [29] studies showed that women were more likely to have diabetes mellitus and hypertension than men with ischemic stroke.

In our results, there were gender differences for mortality, recurrence rate and dependency rate at 3,6, and 12 months after stroke onset. After adjusting for the confounders, the gender differences disappeared for mortality and recurrence rate. There were no gender differences for dependency rates at 3 and 6 months. However, at 12 months after stroke onset, women were still more disabled than men.

Many reports from different countries have assessed sex differences on mortality in stroke patients and their results remain surprisingly variable. Some reports showed no difference for mortality after stroke $[8,9,30,31,32]$, some showed that women had a higher mortality than men $[4,6]$, and others showed a lower mortality in women $[10,11,12,21,25]$. Before adjusting for the confounders, our study showed a higher mortality in women than that in men. However, the difference was not significant after controlling potential confounding factors such as age, history of diabetes, and so on. This suggests that a higher mortality in poststroke women is largely attributed to their older age, more prestroke dependency, severer neurological defect, more cardiovascular risk factors, and a higher pneumonia complication, but not their gender. The Korean Stroke Registry [21], which included 18,634 ischemic stroke or TIA patients admitted within 7 days after symptom onset, showed the similar results as our study at 14-

Table 3. Clinical outcomes at 3, 6, and 12 months after ischemic stroke.

\begin{tabular}{|c|c|c|c|c|c|c|c|c|c|}
\hline \multirow{2}{*}{ Outcomes* } & \multicolumn{3}{|l|}{3 months } & \multicolumn{3}{|l|}{6 months } & \multicolumn{3}{|l|}{12 months } \\
\hline & Women & Men & $P$ Value & Women & Men & $P$ Value & Women & Men & $P$ Value \\
\hline $\begin{array}{l}\text { Mortality } \\
\text { (n/N, \%) }\end{array}$ & $\begin{array}{l}499 / 4,437 \\
(11.3 \%)\end{array}$ & $538 / 7,113(7.6 \%)$ & $<0.001$ & $\begin{array}{l}639 / 4,424 \\
(14.4 \%)\end{array}$ & $685 / 7,078(9.7 \%)$ & $<0.001$ & $\begin{array}{l}789 / 4,442 \\
(17.8 \%)\end{array}$ & $\begin{array}{l}875 / 7,118 \\
(12.3 \%)\end{array}$ & $<0.001$ \\
\hline $\begin{array}{l}\text { Recurrence } \\
\text { rate }(\mathrm{n} / \mathrm{N}, \%)\end{array}$ & $\begin{array}{l}641 / 4,442 \\
(14.4 \%)\end{array}$ & $853 / 7,118(12.0 \%)$ & ) $<0.001$ & $\begin{array}{l}804 / 4,437 \\
(18.1 \%)\end{array}$ & $\begin{array}{l}1,044 / 7,103 \\
(14.7 \%)\end{array}$ & $<0.001$ & $\begin{array}{l}883 / 4,442 \\
(19.9 \%)\end{array}$ & $\begin{array}{l}1,167 / 7,118 \\
(16.4 \%)\end{array}$ & $<0.001$ \\
\hline $\begin{array}{l}\text { Dependency } \\
\text { rate }(\mathrm{n} / \mathrm{N}, \%)\end{array}$ & $\begin{array}{l}1,345 / 3,943 \\
(34.1 \%)\end{array}$ & $\begin{array}{l}1,607 / 6,580 \\
(24.4 \%)\end{array}$ & $<0.001$ & $\begin{array}{l}1,169 / 3,803 \\
(30.7 \%)\end{array}$ & $\begin{array}{l}1,440 / 6,433 \\
(22.4 \%)\end{array}$ & $<0.001$ & $\begin{array}{l}1,049 / 3,653 \\
(28.7 \%)\end{array}$ & $\begin{array}{l}1,192 / 6,243 \\
(19.1 \%)\end{array}$ & $<0.001$ \\
\hline
\end{tabular}

* Mortality was defined as the cumulative death rate at 3,6, and 12 months from the onset of stroke for any reason of death. Recurrence was defined as the aggravated primary neurological deficit, new signs, or rehospitalization with the diagnosis of ischemic stroke, intracerebral hemorrhage, or subarachnoid hemorrhage. Dependency was defined as the modified Rankin Scale scores of 3-5. The recurrence and dependency rates were defined as the cumulative rates at 3, 6 , and 12 months after ischemic stroke, respectively.

doi:10.1371/journal.pone.0056459.t003 
Table 4. Multivariate logistic regression analysis for confounders and outcomes at 12 months after stroke onset.

\begin{tabular}{|c|c|c|c|}
\hline Variables & Mortality OR(95\%Cl) & Recurrence rate $\mathrm{OR}(95 \% \mathrm{Cl})$ & Dependency rate OR(95\%CI) \\
\hline Age & $1.05(1.04-1.05)$ & $1.02(1.01-1.02)$ & $1.04(1.04-1.05)$ \\
\hline Pre-stroke dependency & $1.49(1.18-1.88)$ & $1.65(1.36-2.01)$ & $2.13(1.69-2.67)$ \\
\hline History of smoking & $0.87(0.72-1.04)^{*}$ & $0.93(0.81-1.07)^{*}$ & $0.74(0.64-0.86)$ \\
\hline History of diabetes & $1.30(1.09-1.54)$ & $1.26(1.08-1.47)$ & $1.42(1.19-1.69)$ \\
\hline History of dyslipidemia & $0.55(0.41-0.75)$ & $0.92(0.74-1.13)^{*}$ & $0.89(0.72-1.11)^{*}$ \\
\hline History of coronary heart disease & $0.96(0.77-1.20)^{*}$ & $1.20(1.01-1.43)$ & $0.98(0.81-1.20)^{*}$ \\
\hline History of atrial fibrillation & $1.41(1.07-1.89)$ & $1.06(0.81-1.37)^{*}$ & $0.98(0.71-1.37)^{*}$ \\
\hline History of prior stroke & $1.36(1.14-1.62)$ & $1.43(1.24-1.64)$ & $1.31(1.13-1.53)$ \\
\hline Glucose at admission & $1.08(1.05-1.11)$ & $1.02(0.99-1.04)^{*}$ & $1.04(1.01-1.08)$ \\
\hline \multicolumn{4}{|l|}{ NIHSS at admission } \\
\hline$\geq 15$ versus $0-4$ & $5.26(4.02-6.88)$ & $1.79(1.41-2.28)$ & $11.38(8.48-15.27)$ \\
\hline $5-14$ versus $0-4$ & $1.55(1.26-1.90)$ & $1.18(1.01-1.37)$ & $2.97(2.54-3.46)$ \\
\hline \multicolumn{4}{|l|}{ GCS at admission } \\
\hline $3-8$ versus 15 & $2.54(1.96-3.29)$ & $1.37(1.09-1.73)$ & $1.68(1.29-2.17)$ \\
\hline $9-12$ versus 15 & $1.83(1.42-2.36)$ & $1.30(1.04-1.63)$ & $1.41(1.10-1.79)$ \\
\hline $13-14$ versus 15 & $1.57(1.21-2.05)$ & $1.07(0.85-1.34) *$ & $1.32(1.06-1.64)$ \\
\hline Pneumonia complication & $2.75(2.26-3.35)$ & $1.71(1.43-2.05)$ & $1.86(1.48-2.33)$ \\
\hline New atrial fibrillation complication & $1.45(1.09-1.93)$ & $1.61(1.29-1.99)$ & $1.36(1.04-1.79)$ \\
\hline
\end{tabular}

OR: odds ratio; Cl: confidence interval; NIHSS: National Institute of Health Stroke Scale; GCS: Glasgow Coma Scale.

*Indicated not significantly different.

doi:10.1371/journal.pone.0056459.t004

day and 25-month follow-up before the adjustment. But after adjusting for baseline characteristics of age, histories of hypertension and diabetes mellitus, neurological severity at admission and other factors, women had a greater survival rate than men during both the short- and long-term periods after stroke. The same result was seen in the Demark national registry [25] before the adjustment, which showed a higher crude mortality in women at 30 and 90 days after stroke. But a lower mortality appeared in women after adjusting for the clinical characteristics and other confounders. These different results among the studies probably reflect differences in race/ethnicity and/or follow-up time. Additionally, whether controlling the potential confounders and/ or what kind of the confounding factors were controlled also resulted in different results in mortality after stroke.

Despite the fact that a few studies have been done to find the gender differences in functional outcomes after stroke, almost all the published studies from Europe, North America, and Asia have shown poorer functional outcomes in women than those in men $[8,9,13,21,22,23,24,26,32,33,34]$. Di Carlo A [32] and his colleagues found that women were more disabled and more dependent than men at 3 months after stroke. Their data were from a large registry of multicentres in many countries in Europe and all types of stroke were included. The Rankin Scale was used to evaluate the outcome of handicap at 3-month follow-up and it

Table 5. Unadjusted and adjusted OR of outcomes at 3, 6, and 12 months for women to men.

\begin{tabular}{llll}
\hline Outcomes & & & \\
\hline Mortality & Unadjusted OR (95\%Cl) & Adjusted OR (95\%Cl) * \\
& at 3 months & $1.55(1.36-1.76) \dagger$ & $1.06(0.91-1.24)$ \\
at 6 months & $1.58(1.41-1.77) \dagger$ & $1.10(0.95-1.27)$ \\
Recurrence rate & at 12 months & $1.54(1.39-1.71) \dagger$ & $1.09(0.95-1.24)$ \\
& at 3 months & $1.24(1.11-1.38) \dagger$ & $1.03(0.91-1.16)$ \\
\hline Dependency rate & at 6 months & $1.28(1.16-1.42) \dagger$ & $1.06(0.95-1.19)$ \\
& at 12 months & $1.26(1.15-1.39) \dagger$ & $1.05(0.94-1.17)$ \\
& at 3 months & $1.60(1.47-1.75) \dagger$ & $1.15(0.99-1.34)$ \\
\hline & at 6 months & $1.54(1.41-1.68) \dagger$ & $1.13(0.97-1.31)$ \\
\hline & at 12 months & $1.71(1.55-1.88) \dagger$ & $1.24(1.06-1.45) \dagger$
\end{tabular}

OR: odds ratio.Cl: confidence interval.

*The adjusted risk factors are age, history of diabetes, history of stroke, pre-stroke dependency, stroke severity and blood glucose at admission, and the complications of pneumonia and new atrial fibrillation. ${ }^{\top} \mathrm{P}<0.05$.

doi:10.1371/journal.pone.0056459.t005 
was significantly higher in women than that in men $(2.6 \pm 1.5$ versus 2.2 $\pm 1.5, P<0.001$; Mann-Whitney test). In a Paul Coverdell National Acute Stroke Registry (PCNASR, a statewide stroke registry in America), women were less likely to achieve activities of daily living independence (defined as the Barthel Index $\geq 95$ ) even after adjustment for potential confounders (adjusted OR: 0.37, 95\% CI: 0.19 to 0.87 ), and the researchers also reported the lower least-squares means Stroke-Specific Quality of Life scores in women than that in men [33]. The results from the Registry of the Canadian Stroke Network revealed that women had a slightly worse functional status at 6 months after stroke than men, when they were measured by the Stroke Impact Scale-16 [9]. Our findings are consistent with these reports of gender differences in functional outcomes although we used different measurements to estimate the outcomes and followed patients longer (one year). It has not yet been fully explained why women have poorer functional outcomes. Reeves et al [35] considered the fact that women were more likely to be widowed and had less social support than men might be the reasons for women's poor functional outcomes. However, in our study, the social factors, such as living arrangement, marriage, education, and health insurance, had no significant impact on mortality, recurrence rate, and dependency rate after a multivariate regression analysis. These social factors may prevent women from having good functional outcomes, but their influence is too small to be emphasized when compared with age, stroke severity, history of diabetes mellitus, and pre-stroke function.

Recurrence at 3, 6, and 12 months were similar to death outcome in this study. Nevertheless, in Japan, Fukuda et al [36] found no gender differences on stroke recurrence rate at 1 and 5 years even before controlling potential confounders.

Interestingly, the history of diabetes not hypertension had significant influence on mortality, recurrence rate, and dependency rate in our study. Data from Qian et al [37] and Kong et al [13] confirmed that the post-stroke patients with diabetes mellitus would have poorer outcomes. Although some of previous studies showed that women were less likely to have treatment of thrombolysis than men $[7,21,38,39,40]$, our results showed no gender difference for this treatment, which is consistent with other previous reports $[6,9,41]$. The similar time to arrive at the hospitals between women and men (in three hours after symptom onset) might be the reason for this point in our study.

\section{Strengths and limitations}

This study is a part of the largest population-based prospective stroke registry in China. The completed data with one-year followup have enabled us to investigate the relationship between gender differences and prognoses in post-stroke patients in China, which has not been well-explored previously. However, there are some limitations in this study. Firstly, the enrolled patients were restricted to those from the teaching hospitals or higher level non-teaching hospitals in urban areas of China, not recruiting community-level clinic centers, particularly not covering rural hospitals, where there are high stroke incidences and poor managements. Secondly, regarding risk factors for stroke in

\section{References}

1. Johnston SC, Mendis S, Mathers CD (2009) Global variation in stroke burden and mortality: estimates from monitoring, surveillance, and modelling. Lancet Neurol 8: 345-354.

2. Chen Z (Peking Union Medical College Press of China, Beijing,2008) Report of the third national death cause retrospective investigation.

3. Petrea RE, Beiser AS, Seshadri S, Kelly-Hayes M, Kase CS, et al. (2009) Gender Differences in Stroke Incidence and Poststroke Disability in the Framingham Heart Study. Stroke 40: 1032-1037. women, in addition to common classic risk factors, there are some specific risk factors that may be related to the prognoses of stroke, including pregnancy, delivery, childbearing, puerperium, oral contraceptives, migraine with aura, and hormone replacement therapy after menopause. These specific factors among the specific age groups have remained unclear in the study, which limits the underlying causes of findings in the specific age subgroups and needs further investigation. Thirdly, many patients with ischemic stroke $(2,966$ from 14,526$)$ were excluded that might bias the results. Although there was no significant difference in gender between the enrolled and excluded patients, the excluded patients were younger and less likely to have the risk factors of ischemic stroke. Therefore, the outcomes of the excluded patients might be better than those of the enrolled patients. Fourthly, we did not analyze other types of stroke although their data were collected in the GNSR. In the future, studies are needed to explore the gender differences on prognoses in the patients with other types of stroke, such as hemorrhagic stroke. Fifthly, we followed up patients by telephone not by face to face interview because too many patients were enrolled in this large registry. Thus, the detailed data of stroke recurrence could not be collected in this study and the accuracy of these self-reported events needs to be evaluated. Finally, the Cox proportional hazard regression model may be properer than the logistic regression model to analyze data in our study. However, the precise follow-up data about death or recurrence time of patients were not collected during the telephone follow-ups. Although we used the logistic regression model to analyze data, which could not identify the correlation between the prognostic factors and time, it could still determine the impact from the risk factors on patients' prognoses after ischemic stroke during the follow-up time.

\section{Conclusions}

Our present study indicates that women have many differences in clinical characteristics after ischemic stroke when compared with men in China. The crude mortality, recurrence rate, and dependency rate at 3, 6, and 12 months are higher in women than those in men after ischemic stroke, but the adjustments for the confounders eliminate those differences except for that the 12month dependency rate is still higher in women. Future research should focus on exploring the reasons for this difference in dependency outcome.

\section{Acknowledgments}

We thank all participating hospitals, colleagues, nurses, imaging and laboratory technicians for the GNSR.

\section{Author Contributions}

Conceived and designed the experiments: CW YW. Performed the experiments: ZW JL XZ YW. Analyzed the data: GL AW HL. Contributed reagents/materials/analysis tools: XY. Wrote the paper: ZW JL.

4. Lewsey JD, Gillies M, Jhund PS, Chalmers JWT, Redpath A, et al. (2009) Sex Differences in Incidence, Mortality, and Survival in Individuals With Stroke in Scotland, 1986 to 2005. Stroke 40: 1038-1043.

5. Turaj W, Slowik A, Wnuk M, Szczudlik A (2009) Gender-Related Differences in Diagnostic Evaluation and Outcome of Ischemic Stroke in Poland. Stroke 40: 980-982. 
6. Eriksson M, Glader EL, Norrving B, Terent A, Stegmayr B (2008) Sex Differences in Stroke Care and Outcome in the Swedish National Quality Register for Stroke Care. Stroke 40: 909-914.

7. Reid JM, Dai D, Gubitz GJ, Kapral MK, Christian C, et al. (2008) Gender differences in stroke examined in a 10-year cohort of patients admitted to a Canadian teaching hospital. Stroke 39: 1090-1095.

8. Roquer J, Campello AR, Gomis M (2003) Sex differences in first-ever acute stroke. Stroke 34: 1581-1585.

9. Kapral MK, Fang J, Hill MD, Silver F, Richards J, et al. (2005) Sex differences in stroke care and outcomes: results from the Registry of the Canadian Stroke Network. Stroke 36: 809-814.

10. Sheikh K, Bullock CM (2007) Effect of measurement on sex difference in stroke mortality. Stroke 38: 1085-1087.

11. Holroyd-Leduc JM, Kapral MK, Austin PC, Tu JV (2000) Sex differences and similarities in the management and outcome of stroke patients. Stroke 31: 18331837.

12. Benatru I, Rouaud O, Durier J, Contegal F, Couvreur G, et al. (2006) Stable stroke incidence rates but improved case-fatality in Dijon, France, from 1985 to 2004. Stroke 37: 1674-1679.

13. Kong F-Y, Tao W-D, Hao Z-L, Liu M (2010) Predictors of One-Year Disability and Death in Chinese Hospitalized Women after Ischemic Stroke. Cerebrovascular Diseases 29: 255-262.

14. Wang Y, Cui L, Ji X, Dong Q, Zeng J, et al. (2011) The China National Stroke Registry for patients with acute cerebrovascular events: design, rationale, and baseline patient characteristics. Int J Stroke 6: 355-361.

15. (1989) Stroke-1989. Recommendations on stroke prevention, diagnosis, and therapy. Report of the WHO Task Force on Stroke and other Cerebrovascular Disorders. Stroke 20: 1407-1431.

16. Dong K (2009) Medical insurance system evolution in China. China Econ Rev 20: 591-597.

17. Liu Y (2004) Development of the rural health insurance system in China. Health Policy Plan 19: 159-165.

18. Liu Y (2002) Reforming China's urban health insurance system. Health Policy Plan 60: 133-150.

19. Brott T, Adams HP Jr, Olinger CP, Marler JR, Barsan WG, et al. (1989) Measurements of acute cerebral infarction: a clinical examination scale. Stroke 20: $864-870$.

20. Teasdale G, Jennett B (1974) Assessment of coma and impaired consciousness. A practical scale. Lancet 2: 81-84.

21. Oh M-S, Yu K-H, Roh J-K, Lee B-C (2009) Gender Differences in the Mortality and Outcome of Stroke Patients in Korea. Cerebrovascular Diseases 28: 427434.

22. Niewada M, Kobayashi A, Sandercock PA, Kaminski B, Czlonkowska A (2005) Influence of gender on baseline features and clinical outcomes among 17,370 patients with confirmed ischaemic stroke in the international stroke trial. Neuroepidemiology 24: 123-128.

23. Caso V, Paciaroni M, Agnelli G, Corea F, Ageno W, et al. (2010) Gender differences in patients with acute ischemic stroke. Womens Health (Lond Engl) 6: $51-57$.

24. Silva GS, Lima FO, Camargo EC, Smith WS, Lev MH, et al, (2010) Gender differences in outcomes after ischemic stroke: role of ischemic lesion volume and intracranial large-artery occlusion. Cerebrovasc Dis 30: 470-475.

25. Palnum KD, Andersen G, Ingeman A, Krog BR, Bartels P, et al. (2009) SexRelated Differences in Quality of Care and Short-Term Mortality Among
Patients With Acute Stroke in Denmark: A Nationwide Follow-Up Study. Stroke 40: 1134-1139.

26. Glader EL, Stegmayr B, Norrving B, Terent A, Hulter-Asberg K, et al. (2003) Sex differences in management and outcome after stroke: a Swedish national perspective. Stroke 34: 1970-1975.

27. Yang ZJ, Liu J, Ge JP, Chen L, Zhao ZG, et al. (2012) Prevalence of cardiovascular disease risk factor in the Chinese population: the 2007-2008 China National Diabetes and Metabolic Disorders Study. Eur Heart J 33: 213 220.

28. Zhang B, Pu S, Zhang W, Yang N, Shen G, et al. (2011) Sex differences in risk factors, etiology, and short-term outcome of cerebral infarction in young patients. Atherosclerosis 216: 420-425.

29. Huang Y, Wang J-G, Wei JW, Headley AP, Wong LKS, et al. (2010) Research: Age and gender variations in the management of ischaemic stroke in China. International Journal of Stroke 5: 351-359.

30. Palm F, Urbanek C, Wolf J, Buggle F, Kleemann T, et al. (2011) Etiology, Risk Factors and Sex Differences in Ischemic Stroke in the Ludwigshafen Stroke Study, a Population-Based Stroke Registry. Cerebrovasc Dis 33: 69-75.

31. Kapral MK, Degani N, Hall R, Fang J, Saposnik G, et al. (2011) Gender differences in stroke care and outcomes in Ontario. Womens Health Issues 21: 171-176.

32. Di Carlo A, Lamassa M, Baldereschi M, Pracucci G, Basile AM, et al. (2003) Sex differences in the clinical presentation, resource use, and 3-month outcome of acute stroke in Europe: data from a multicenter multinational hospital-based registry. Stroke 34: 1114-1119.

33. Gargano JW, Reeves MJ (2007) Sex differences in stroke recovery and strokespecific quality of life: results from a statewide stroke registry. Stroke 38: 25412548 .

34. Saji N, Shimizu H, Kawarai T, Tadano M, Kita Y, et al. (2011) Clinical features of a first-ever lacunar infarction in Japanese patients: poor outcome in females. J Stroke Cerebrovasc Dis 20: 231-235.

35. Reeves MJ, Bushnell CD, Howard G, Gargano JW, Duncan PW, et al. (2008) Sex differences in stroke: epidemiology, clinical presentation, medical care, and outcomes. Lancet Neurol 7: 915-926.

36. Fukuda M, Kanda T, Kamide N, Akutsu T, Sakai F (2009) Gender Differences in Long-term Functional Outcome after First-ever Ischemic Stroke. Internal Medicine 48: 967-973.

37. Jia Q Zhao X, Wang C, Wang Y, Yan Y, et al. (2011) Diabetes and poor outcomes within 6 months after acute ischemic stroke: the China National Stroke Registry. Stroke 42: 2758-2762.

38. Gargano JW, Wehner S, Reeves M (2008) Sex differences in acute stroke care in a statewide stroke registry. Stroke 39: 24-29.

39. Deng YZ, Reeves MJ, Jacobs BS, Birbeck GL, Kothari RU, et al. (2006) IV tissue plasminogen activator use in acute stroke: experience from a statewide registry. Neurology 66: 306-312.

40. Bateman BT, Schumacher HC, Boden-Albala B, Berman MF, Mohr JP, et al (2006) Factors associated with in-hospital mortality after administration of thrombolysis in acute ischemic stroke patients: an analysis of the nationwide inpatient sample 1999 to 2002. Stroke 37: 440-446.

41. Allen NB, Myers D, Watanabe E, Dostal J, Sama D, et al. (2009) Utilization of intravenous tissue plasminogen activator for ischemic stroke: are there sex differences? Cerebrovasc Dis 27: 254-258. 